\title{
Manufactura esbelta: una revisión sistemática en la industria de alimentos
}

\author{
Cynthia Cuggia-Jiménez ${ }^{1}$, Erick Orozco-Acosta ${ }^{1 *}$ y Darwin Mendoza-Galvis ${ }^{2}$ \\ (1) Universidad Simón Bolívar, Facultad de Ingenierías, Barranquilla, Colombia. \\ (correo-e: cynthiacuggia@hotmail.com; eorozco15@unisimonbolivar.edu.co) \\ (2) Universidad de Santander, Facultad de Ingeniería Industrial, Grupo de Investigación Nuevas Tecnologías-UDES, \\ Cra. 6 No. 14-27, Valledupar-Colombia. (correo-e: dar.mendoza@mail.udes.edu.co)
}

*Autor a quien debe ser dirigida la correspondência.

Recibido Mar. 30, 2020; Aceptado Jun. 1, 2020; Versión final Jul. 22, 2020, Publicado Oct. 2020

\section{Resumen}

Este artículo describe las tendencias de estudio de manufactura esbelta mediante una revisión sistemática de la literatura entre los años 2015 y 2019 de las principales bases de datos bibliográficas. Se recuperaron 3776 artículos y se aplicó un análisis de conglomerado bibliométrico por tema y año con los metadatos y la herramienta VOSviewer. Los resultados indican que la base de datos con mayor número de publicaciones es Science Reseach. El 36\% del total de los artículos recuperados son de países de oriente, los cuales abordan la eficiencia de procesos productivos y la productividad. Se proporciona un análisis de la implementación de la filosofía de manufactura esbelta, así como un resumen de las herramientas utilizadas en empresas del sector de alimentos. Finalmente, se propone un marco general que resume las tendencias en la temática de manufactura esbelta a partir de la revisión de literatura desarrollada en el presente trabajo.

Palabras clave: manufactura esbelta; industria alimentos; revisión sistemática, análisis bibliométrico

\section{Lean manufacturing: a systematic review in the food industry}

\begin{abstract}
This paper describes the study trends of lean manufacturing at a national and international level through a systematic review of the scientific literature between 2015 and 2019. A total of 3776 research articles were retrieved from the main bibliographic databases. Metadata and VOSviewer were used to perform a bibliometric cluster analysis by topic and year. The results indicated that the database with the largest number of publications was Science Research. In addition, $36 \%$ of all the research articles retrieved were from Asian countries and they addressed efficiency of production processes and productivity. The present article provided an analysis of the implementation of the lean manufacturing philosophy and a summary of the tools used in companies in the food sector to generate inputs that promote productivity. A general framework is proposed that summarizes the trends in lean manufacturing from the literature review performed here.
\end{abstract}

Keywords: lean manufacturing; food industry; systematic review, bibliometric analysis 


\section{INTRODUCCIÓN}

El término Manufactura Esbelta se menciona por primera vez al inicio de la década de los 90's en el libro "The machine that changed the world" (Womack et al, 1991), como resultado de investigaciones referentes a estudios comparativos de prácticas de producción alrededor del mundo. A partir de este hito histórico el concepto se ha extendido en sus aplicaciones en diversas industrias y es reconocido mundialmente (Viteri et al., 2016). Sin embargo, su origen se viene gestando desde la primera mitad del siglo XX en los sectores como la industria automotriz (Caso del sistema de producción Toyota) donde se llevaba a cabo la producción en masa, que es su principio básico (Rajadell-Carreras y Sánchez, 2010). La segunda guerra mundial, genera avances en la administración de operaciones en campos como la computación digital, programación lineal, procesos estocásticos, el estudio continuo del trabajo y la integración de las operaciones (Kumar y Suresh, 2009). Esto generó que muchas industrias crecieran, pero al mismo tiempo, los procesos aumentaran su complejidad combinados por fenómenos macroeconómicos (como las crisis o depresiones económicas), los costos fijos de producción aumentaron y disminuyó la rentabilidad, provocada básicamente por los despilfarros en los recursos de los procesos productivos (Chase et al, 2009).

Muchas son las definiciones y autores que han aportado al estudio de la manufactura esbelta, entre las que se destacan, la definen como un proceso continuo y sistemático de identificación y eliminación del desperdicio o excesos, entendiendo como exceso toda aquella actividad que no agrega valor en un proceso, pero sí costo y trabajo (Socconini, 2008). Asimismo, desde una perspectiva más gerencial, se debe entender que esta herramienta es el esfuerzo incansable y continuo para crear empresas más efectivas, innovadoras y eficientes (Bodek, 2010). Para tal efecto, tiene como objetivo realizar la producción con cero defectos, reducir costos, satisfacer las solicitudes de los clientes en el momento y la cantidad deseada, no tener inventarios en exceso y realizar mejoras continuamente eliminando los desechos (Arslankaya y Atay, 2015). Esta herramienta posee muchas técnicas para su desarrollo práctico (Tapia et al., 2017); por lo tanto, la investigación estuvo sustentada o desarrollada por las siguientes: JIT (Just In Time) que se enfoca en los inventarios que van quedando en cada fase del proceso, SMED (Single Minute Exchange of Die - reducción de los desperdicios) implementado en los tiempos de cambio entre tipo de producto, 5S para efectos del orden y aseo en puestos de trabajo (Baena et al., 2008), TPM (Mantenimiento Productivo Total) para el mantenimiento de las máquinas (González-Correa, 2007) y Heijunka (nivelación de la producción) basado en la forma de programación de la producción en la empresa.

En el contexto colombiano, las micro, pequeñas y medianas empresas (MiPymes) son representantes trascendentales la reactivación de la economía, la producción nacional y el progreso competitivo del país. Según, el Departamento Administrativo Nacional de Estadística (DANE), las MiPymes ayudan con el 35\% del Producto Interno Bruto (PIB), generan el $80 \%$ del empleo y constituyen el $90 \%$ del sector productivo de Colombia; asimismo se evidencia un gran aporte en generación de empleo en el país (Hernandez, 2018). Adicionalmente, la dinámica sectorial no es ajena a las cifras macroeconómicas. Según la Agencia de Promoción de Inversiones en Bogotá (2018), el sector de alimentos y bebidas alcanzó ventas por 13.200 millones de dólares en 2017 y tuvo más presentación en las industrias de molinería, panadería y repostería, con un $31 \%$, seguido de los lácteos, harinas, confitería, snacks, frutas, legumbres, aceites, salsas, cárnicos, helados y postres (CVN, 2018). Tomando como referencia dos ciudades como Valledupar y Barranquilla, se pueden establecer que existen 130 empresas panaderas legalmente constituidas (Cámara de Comercio de Valledupar, 2019); y en Barranquilla, existen 468 panaderías legalmente constituidas (Cámara de Comercio de Barranquilla, 2019); del porcentaje total entre las dos ciudades, el $100 \%$ corresponden a MiPymes. Específicamente en Colombia, Bogotá es la ciudad que más panaderías alberga en el país, con cerca de 7.000 puntos, seguido de Cali con 2.165, Medellín 1.532, Barranquilla 565 y Bucaramanga con 466. Con base en estas cifras, existe una panadería por cada 1.100 habitantes de los estratos sociales 1,2 y 3 , donde se presenta la mayor concentración de la demanda.

Considerando la representatividad del sector alimentario es fundamental favorecer las condiciones de competitividad de mismo. En este sentido, dentro de los factores que explican el aumento de la productividad de las empresas, el $65 \%$ corresponde a los esfuerzos internos que puede hacer cada compañía, como las ganancias por posicionamiento de productos y obtención de mayor participación en el mercado, y la mejora de procesos productivos o las prácticas gerenciales (Consejo Privado de Competitividad, 2017). No obstante, las empresas, especialmente las Pymes, enfrentan diversas situaciones que restringen su productividad, tales como la dificultad para lograr el control de los procesos, la inexistencia estándares de calidad implementación, la generación de desperdicios en tiempo de producción y la inadecuada utilización de los equipos (Velásquez, 2003). En consecuencia, en la gran mayoría de empresas, es poco común la implementación exitosa de prácticas de manufactura esbelta. Una de las causas es la carencia de metodologías de implementación práctica; otra es los tiempos de implementación, lo que puede implicar costos agregados que impactan los presupuestos de las empresas; por tal razón es tan mínimo el nivel de implementación de esta herramienta (Sarria et al., 2017). Este hecho puede tener causas asignables en el panorama de corto plazo de planificación 
de operaciones de las empresas o en el día a día absorbente de las industrias que genera que se planifique casi que tomando como referencia el desperdicio con pocas acciones para mitigarlo.

Desde esta perspectiva, es relevante establecer acciones encaminadas al mejoramiento del sector panadero, mediante el desarrollo de prácticas efectivas de producción, ámbito en el cual la manufactura esbelta representa un enfoque de especial interés, debido a su enfoque en la eliminación de desperdicios. Para tal fin, el compromiso gerencial, el liderazgo y el apoyo económico se constituyen como elementos cruciales para la implementación de las técnicas de manufactura esbelta, considerando que en la industria panificadora, la aplicación de estas técnicas ha venido consolidándose producto de las demandas de los consumidores y los competidores (Quesada y Arrieta, 2019). Adicionalmente, la literatura reconoce la importancia de la investigación relacionada con la implementación de herramientas de manufactura esbelta en la industria panadera debido a la importancia del sector para la economía (Ferreira et al., 2017).

En referencia a los avances de la teoría de manufactura esbelta, la investigación de Psomas y Antony, (2019) destaca, a partir de una revisión de la literatura entre el año 2005 y 2016, que las contribuciones a la literatura de manufactura esbelta están fragmentadas y muestran algunas limitaciones importantes. En este sentido, nuestro documento presenta una revisión de la literatura reciente sobre el tema, y propone un marco conceptual que sintetiza las tendencias recientes del conocimiento sobre manufactura esbelta. Adicionalmente, el enfoque de la revisión en el sector alimentos, representa una contribución a la temática analizada. De igual modo, este trabajo aporta al conocimiento de este tema, ya que hace una recopilación de las herramientas y aplicaciones existentes, identificando los aspectos relevantes para su implementación.

Adicionalmente, existe una escasa evidencia de revisión de literatura previas en la temática de manufactura esbelta en el sector alimentos, puntualmente, para efectos de la realización de este trabajo se identificaron dos antecedentes al respecto, desarrollados por los autores Stevenson y Jain (2005) y Costa et al. (2018). En consecuencia, este articulo presenta una contribución significativa al conocimiento de manufactura esbelta y su implementación en este tipo de industrias. Al respecto, la revisión realizada por Costa et al. (2018) destaca la necesidad de generar un mayor número de investigaciones para mejorar la comprensión de las prácticas de manufactura esbelta que se implementan en la industria de alimentos y determinar las estrategias adecuadas para implementar esas iniciativas.

Así, este trabajo presenta las tendencias de estudio de manufactura esbelta a nivel nacional e internacional a través de una revisión sistemática de la literatura científica del tema de estudio. Se trabajó con ecuaciones de búsqueda en bases de datos arbitradas por pares para observar la evolución y estructura de forma agregada de la investigación en torno a prácticas exitosas en empresas del sector de alimentos como las panaderías y afines. El documento consta de una metodología que esboza el esquema general de trabajo en la cual se fundamenta la revisión. Seguidamente, en los resultados se tiene el procesamiento de la información primaria de los metadatos de las publicaciones importadas en archivos planos por las bases de datos. Por último, se detallan las conclusiones de la revisión.

\section{METODOLOGÍA}

Para la revisión sistemática, se tomaron como referencia artículos científicos en los últimos 5 años comprendidos entre 2015 y 2019. Las bases de datos usadas en el estudio fueron: SciELO, Science Direct, Scopus, Dialnet, Springer Link, ERIC y Science Research. Se establecieron las siguientes ecuaciones de búsqueda (usando operadores Booleanos): "Lean Manufacturing", "Lean Manufacturing AND food industry", "Lean Manufacturing AND bakery", "Just in time AND bakery", "Just in time AND food industry" y "SMED AND food industry". Con estos criterios, se obtuvieron un total de 3776 artículos (Tabla 1), mediante el cual se establecen las tendencias de las publicaciones de literatura científica de manufactura esbelta.

Con los documentos encontrados, se generó de un archivo .RIS (Sistema de información de investigación, siglas en inglés) que es un archivo que contiene metadatos de publicaciones científicas como nombre de los autores, nombre de la revista, país de filiación de los autores, institución de filiación, palabras claves del documento, entre otros. A partir de este, se realizó un aplicó un análisis de conglomerado bibliométrico y se optó por el método de conteo completo con un número mínimo de concurrencia de 2 palabras claves, con normalización, uniendo los grupos de pequeñas frecuencias y con un máximo de 1000 iteraciones. Para este, se usó la herramienta VOSviewer.

Seguidamente, para analizar en profundidad la información publicada en los sectores como alimentos y panaderías, a los documentos recopilados se le aplicaron los siguientes criterios de inclusión: en primer lugar, se trabajó solamente con artículos de investigación entre todos los tipos de trabajos que se publican en las revistas científicas, por lo que se extraen 578 documentos. Luego, tomando como referencia la coherencia entre el contenido del título y el tema objetivo de la revisión se descartan 1975 trabajos. 
Tabla 1: Evolución temporal de las publicaciones de literatura científica de manufactura esbelta

\begin{tabular}{|c|c|c|c|c|c|c|}
\hline \multirow{2}{*}{ Base de datos } & \multirow{2}{*}{ Ecuación de búsqueda } & \multicolumn{5}{|c|}{ Año } \\
\hline & & 2015 & 2016 & 2017 & 2018 & 2019 \\
\hline SciELO & "Lean Manufacturing" & 6 & 9 & 9 & 3 & 2 \\
\hline \multirow{4}{*}{$\begin{array}{l}\text { Science } \\
\text { Direct }\end{array}$} & "Lean Manufacturing" & 167 & 172 & 194 & 213 & 108 \\
\hline & "Lean Manufacturing" AND food industry & 32 & 28 & 42 & 48 & 23 \\
\hline & "Lean Manufacturing" AND bakery & 1 & 2 & 2 & 3 & 1 \\
\hline & "SMED" AND food industry & 8 & 7 & 25 & 18 & 13 \\
\hline \multirow{4}{*}{ SCOPUS } & "Lean Manufacturing" & 39 & 37 & 49 & 86 & 24 \\
\hline & "Lean Manufacturing" AND food industry & 2 & 6 & 5 & 6 & 2 \\
\hline & "Lean Manufacturing" AND bakery & 0 & 0 & 0 & 0 & 1 \\
\hline & "Just in time" AND food industry & 5 & 5 & 3 & 0 & 1 \\
\hline Dialnet & "Lean Manufacturing" & 0 & 2 & 1 & 1 & 0 \\
\hline \multirow{6}{*}{$\begin{array}{l}\text { Springer } \\
\text { Link }\end{array}$} & "Lean Manufacturing" & 47 & 68 & 61 & 62 & 43 \\
\hline & "Lean Manufacturing" AND food industry & 9 & 9 & 14 & 13 & 13 \\
\hline & "Lean Manufacturing" AND bakery & 0 & 0 & 0 & 0 & 1 \\
\hline & "Just in time" AND bakery & 1 & 1 & 1 & 1 & 0 \\
\hline & "Just in time" AND food industry & 45 & 44 & 48 & 59 & 28 \\
\hline & "SMED" AND food industry & 3 & 1 & 7 & 5 & 6 \\
\hline ERIC & "Lean Manufacturing" & 1 & 0 & 3 & 1 & 0 \\
\hline \multirow{3}{*}{$\begin{array}{l}\text { Science } \\
\text { Research }\end{array}$} & "Lean Manufacturing" & 142 & 132 & 197 & 249 & 260 \\
\hline & "Lean Manufacturing" AND bakery & 9 & 20 & 20 & 19 & 52 \\
\hline & "Just in time" AND food industry & 75 & 88 & 145 & 138 & 224 \\
\hline
\end{tabular}

Después, se realiza la revisión del resumen criterio que excluye 931 artículos. Finalmente, se realiza la revisión a texto completo de los documentos, que abordó aspectos como palabras claves, objetivo, diseño, técnicas de análisis de datos, instrumentos de recolección de datos, principales resultados, pregunta de investigación y variables a medir (herramientas de manufactura esbelta). A partir de esto se retiran 270 documentos, para quedar con 22 trabajos ajustados al tema objetivo. La aplicación de los criterios de forma secuencial se puede observar en la Figura 1.

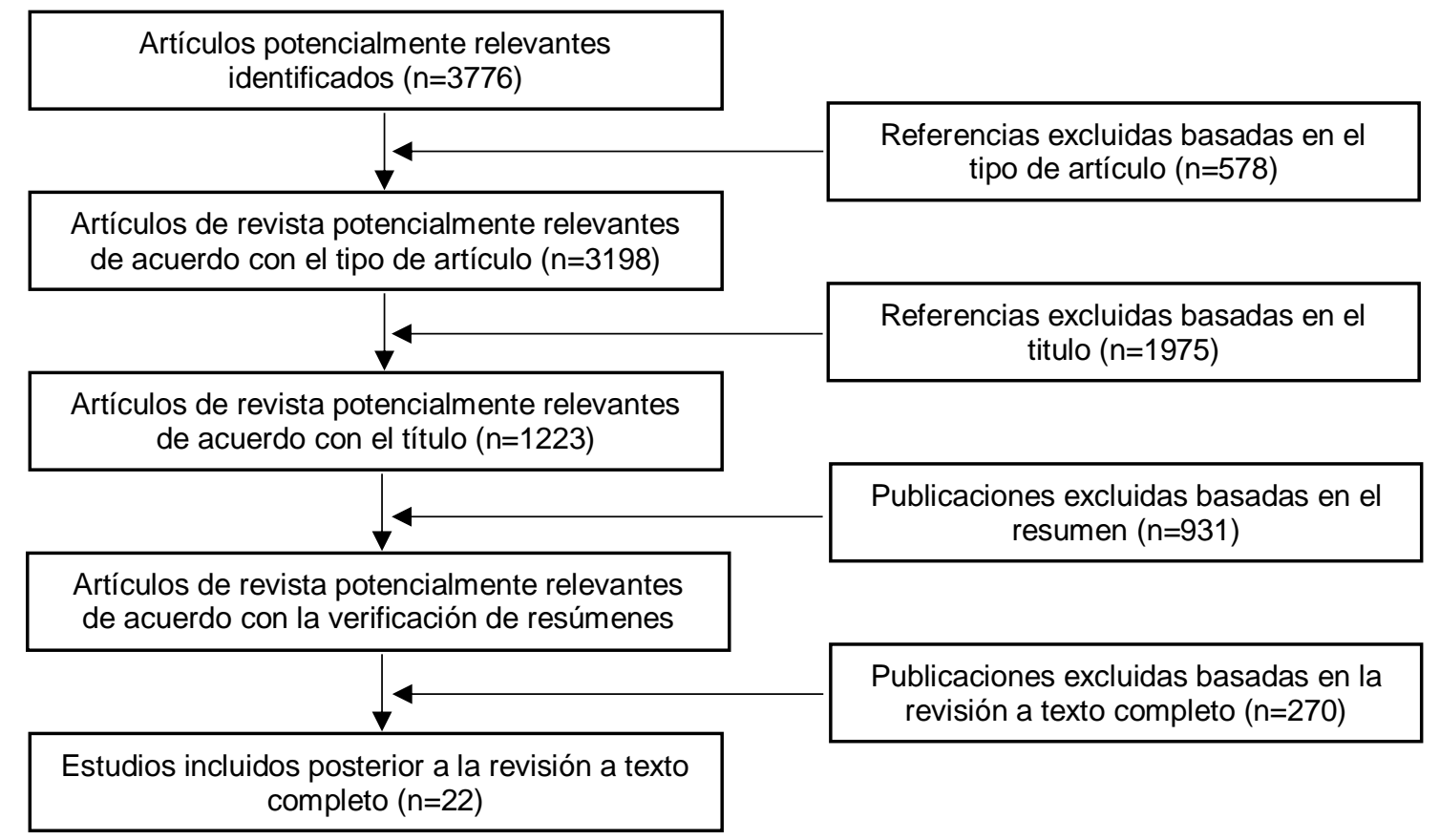

Fig. 1. Flujograma de exclusión de artículos 


\section{RESULTADOS}

En la Figura 2, se puede observar la tendencia del número de publicaciones por año segregadas en series por bases de datos (en las que se encontraron publicaciones en mayor frecuencia), incluyendo una serie "Total". En esta, se puede observar que existe una tendencia creciente en el número de publicaciones entre los años 2015 y 2019 (tomando como referencia el $90.1 \%$ de los documentos), debido a que muestra una pendiente positiva, con un pico de 920 en el 2018. En la tendencia por bases de datos, hay un comportamiento estable en Science Direct y Springer Link, una tendencia a la baja en SCOPUS y al alza de Science Research. Al analizar la participación por base de datos, Science Reseach tiene el $47 \%$ de los trabajos, Science Direct el $30 \%$, Springer Link un $16 \%$ y SCOPUS con un $7 \%$.

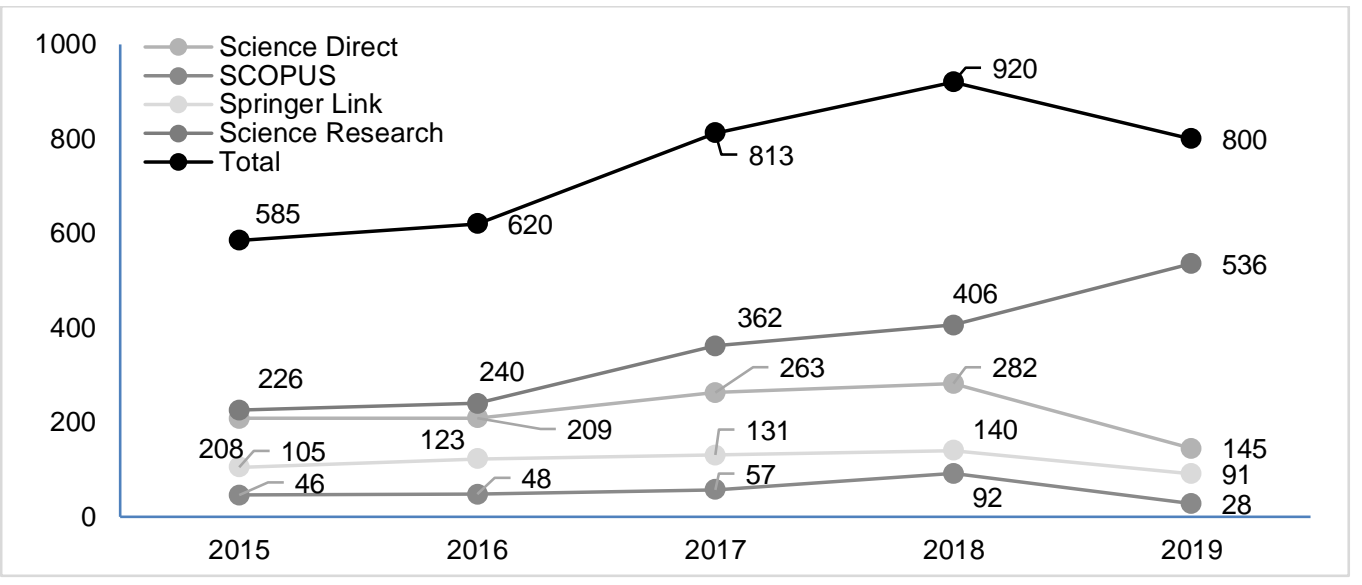

Fig. 2. Evolución en el tiempo de la literatura científica para manufactura esbelta

En la Tabla 2, se muestran los conglomerados resultantes del análisis bibliométrico realizado por la concurrencia de las palabras claves. En total se obtuvieron 88 palabras claves de los artículos analizados; a partir de un número mínimo de 2 concurrencias. Los términos con la mayor fuerza de enlace total de coincidencia fueron: manufactura esbelta, desperdicio y productividad. Se obtuvieron 8 clústeres por las palabras claves más relevantes. La búsqueda por la ventana de tiempo indica que no es un tema reciente y que lleva tiempo implementándose a nivel mundial; ha tomado fuerza entre las empresas, ya que Manufactura esbelta es otra herramienta desarrollada por la por la Compañía Toyota que sirve para mejorar y optimizar los procesos operativos de cualquier compañía industrial, independientemente de su tamaño; el objetivo es minimizar el desperdicio (Padilla, 2010). Luego, tomando como referencia los documentos seleccionados por el flujo de la Figura 1, se tienen los trabajos con sus aportes más importantes (Tabla 3).

Tabla 2: Ítems relevantes por clústeres

\begin{tabular}{|c|c|}
\hline Clúster & İtems \\
\hline 1 & $\begin{array}{l}\text { Montaje, ventaja competitiva, mejora continua, reducción de costo, éxito, tiempo de espera, fabricación, } \\
\text { proceso, producción, seis sigma, Pyme, SMED, VSM y residuos. }\end{array}$ \\
\hline 2 & $\begin{array}{l}\text { Ágil, benchmarking, cuidado de la salud, información, inventario, compromiso de conocimiento, cadena de } \\
\text { suministro, gestión del rendimiento, gestión de riesgos, modelo de ecuaciones estructurales, sustentabilidad. }\end{array}$ \\
\hline 3 & $\begin{array}{l}\text { Agricultura, combustibles alternativos, contaminación del aire, aleaciones binarias, emisión y huella de } \\
\text { carbono, producción más limpia, cambio climático, análisis de costos, eco-eficiencia, análisis económico, } \\
\text { crecimiento económico, gestión energética, medio ambiente, materias primas, industria, análisis del ciclo de } \\
\text { vida, recursos naturales, sostenible. }\end{array}$ \\
\hline 4 & $\begin{array}{l}\text { Cadena de suministro agroalimentario, agroindustria, negocios, competitividad, responsabilidad social } \\
\text { corporativa, transformación digital, eco-innovación, emprendimiento, suministro de alimentos, globalización, } \\
\text { desarrollo industrial, innovación, fabricación, Pyme, grupos de interés, depósito de basura. }\end{array}$ \\
\hline 5 & $\begin{array}{l}\text { Impresión 3D, fabricación aditiva, animales, bacterias, carne de res, bebidas, bio-acumulación, bovinos, } \\
\text { productos lácteos, industria farmacéutica, medioambiental, pescado, productos alimenticios, riesgos de } \\
\text { salud, Justo a tiempo, prácticas de Lean Manufacturing, metabolismo, obesidad, calidad, cumplimiento } \\
\text { normativo, factor de riesgo. }\end{array}$ \\
\hline 6 & $\begin{array}{l}\text { Proceso de jerarquía analítica, evaluación, barreras, conservación, construcción, toma de decisiones, facilita- } \\
\text { dores, teoría de conjuntos difusos, fiabilidad, análisis de riesgo, sostenibilidad social, cadena de suministro. }\end{array}$ \\
\hline 7 & $\begin{array}{l}\text { Estudios de caso, sostenibilidad corporativa, adquisición de datos, estudios empíricos, plan de recursos } \\
\text { empresariales, cadena de suministro de alimentos, gestión industrial, ISO } 14001 \text { y } 9001 \text {, implementación de } \\
\text { lean, investigación de operaciones, gestión de la calidad, Pyme, factores de éxito, cadena de suministro. }\end{array}$ \\
\hline 8 & $\begin{array}{l}\text { Análisis de big data, inteligencia artificial, blockchain, comercio, sistema físico cibernético, análisis de datos, } \\
\text { minería de datos, sistemas embebidos, revoluciones industriales, industria 4.0, información y comunicación, } \\
\text { internet, IOT (internet de las cosas), red neuronal, proceso de innovación, eficiencia de recursos, innovación } \\
\text { sostenible, innovación tecnológica. }\end{array}$ \\
\hline
\end{tabular}


En la Tabla 3 se puede observar que el 36\% (más de 1 de cada 3 ) de los trabajos seleccionados son de países del continente asiático como Indonesia y Malasia. Un 27\% proviene de países de Latinoamérica como Brasil, Colombia, Ecuador y México. Con igual frecuencia, se tienen trabajos de países del continente europeo como Francia, Polonia, Reino Unido y Rumania. Y, el restante 10\% proviene de Qatar y Turquía, que son países de oriente medio que viven un gran auge económico en el sector de alimentos y bebidas por su diversificación en la inversión de capitales.

Tabla 3: Matriz de revisión sistemática

\begin{tabular}{|c|c|c|}
\hline Autor (es) & País & Principales resultados \\
\hline $\begin{array}{l}\text { Quesada y } \\
\text { Arrieta (2019) }\end{array}$ & Colombia & $\begin{array}{l}\text { Las prácticas de Manufactura esbelta aplicadas y destacadas en las panaderías y/o } \\
\text { pastelerías son: poka yoke, kaizen y visual factory. }\end{array}$ \\
\hline $\begin{array}{l}\text { Tapia et al., } \\
\text { (2017) }\end{array}$ & México & $\begin{array}{l}\text { Las } 5 \text { 'S, el VSM, Kaizen, Kanban y TPM son las más utilizadas en el ramo } \\
\text { Manufacturero con un } 9,46 \%, 8.1 \%, 6,75 \%, 5,4 \% \text { y } 4,05 \% \text { respectivamente, y SMED } \\
\text { con un } 4,05 \% \text { y JIT con un } 6,76 \% \text { en el sector Automotriz; caso contrario, las Células } \\
\text { de Manufactura, Heijunka y Andon son las menos utilizadas (en } 1,35 \% \text { ). }\end{array}$ \\
\hline $\begin{array}{l}\text { Sarria et al., } \\
(2017)\end{array}$ & Colombia & $\begin{array}{l}\text { Resalta la importancia de realizar un detallado diagnóstico que permita identificar las } \\
\text { causas que impactan negativamente en el desarrollo del sistema productivo, para así } \\
\text { descubrir y eliminar los desperdicios a través de las diferentes prácticas de } \\
\text { manufactura esbelta. }\end{array}$ \\
\hline $\begin{array}{l}\text { Viteri et al., } \\
\text { (2016) }\end{array}$ & Ecuador & $\begin{array}{l}\text { Al aplicar manufactura esbelta y eliminar diferentes tipos de residuos, se podría } \\
\text { obtener una reducción de } 24 \text { minutos en el ciclo de producción total. Además, es } \\
\text { posible decir que por cada dólar que la compañía gastó en la implementación de la } \\
\text { metodología mencionada, generó un rendimiento de } \$ 0,70 \text {. }\end{array}$ \\
\hline $\begin{array}{l}\text { Borges et al., } \\
(2015)\end{array}$ & Portugal & $\begin{array}{l}\text { Se encontraron varias mejoras en la implementación de los principios y herramientas } \\
\text { de manufactura esbelta que impactan procesos de alimentos y bebidas, en particular } \\
\text { las empresas medianas y grandes. }\end{array}$ \\
\hline $\begin{array}{l}\text { Salem et al., } \\
(2016)\end{array}$ & Qatar & $\begin{array}{l}\text { Las industrias en Qatar deben otorgar más importancia al pensamiento de } \\
\text { manufactura esbelta para avanzar estratégicamente en las eficiencias actuales. }\end{array}$ \\
\hline $\begin{array}{l}\text { Arslankaya y } \\
\text { Atay, (2015) }\end{array}$ & Turquía & $\begin{array}{l}\text { Se logró la continuidad de la producción, reducir el costo de producción, aumentar el } \\
\text { rendimiento y la calidad del producto, aumentar el índice de utilización de la } \\
\text { capacidad, para extender la vida útil de la maquinaria y el equipo, para garantizar la } \\
\text { seguridad del personal y para reducir los costos de mantenimiento y reparación. }\end{array}$ \\
\hline $\begin{array}{l}\text { Munteanu y } \\
\text { Ştefănigă, } \\
(2018)\end{array}$ & Rumania & $\begin{array}{l}\text { Se encontró poca información en el campo de la manufactura esbelta y detalles sobre } \\
\text { el método de producción justo a tiempo. }\end{array}$ \\
\hline $\begin{array}{l}\text { Antosz y } \\
\text { Stadnicka, } \\
(2017)\end{array}$ & Polonia & $\begin{array}{l}\text { Los resultados muestran que muchas pymes desean implementar la filosofía de } \\
\text { manufactura esbelta. Estas empresas quieren mejorar su funcionamiento o se dan } \\
\text { cuenta de la necesidad de eliminar los residuos. }\end{array}$ \\
\hline $\begin{array}{l}\text { Hartini y } \\
\text { Ciptomulyono, } \\
(2015)\end{array}$ & Indonesia & $\begin{array}{l}\text { Existe un impacto positivo de la manufactura esbelta y sostenible en la economía } \\
\text { medio ambiente y sociedad. }\end{array}$ \\
\hline $\begin{array}{l}\text { Widiasih et al., } \\
(2015)\end{array}$ & Indonesia & $\begin{array}{l}\text { Hubo cambios en la magnitud del riesgo de generar desperdicios cuando se } \\
\text { analizaron las interrelaciones entre los procesos. }\end{array}$ \\
\hline $\begin{array}{l}\text { Moeuf et al., } \\
(2016)\end{array}$ & Francia & $\begin{array}{l}\text { Una barrera fuerte en la adopción de la cultura de manufactura esbelta es el proceso } \\
\text { de toma de decisiones que está permeado por fuertes tendencias de resistencia al } \\
\text { cambio. }\end{array}$ \\
\hline $\begin{array}{l}\text { Sousa et al., } \\
(2018)\end{array}$ & Portugal & $\begin{array}{l}\text { Las herramientas de manufactura esbelta son un método poderoso para obtener } \\
\text { rendimientos sólidos sin grandes inversiones. Al aplicar la metodología SMED se } \\
\text { logró reducir el tiempo de inactividad en un } 43 \% \text { en el tiempo de cambio total. }\end{array}$ \\
\hline $\begin{array}{l}\text { Iranmanesh et } \\
\text { al., (2019) }\end{array}$ & Malasia & $\begin{array}{l}\text { La cultura de manufactura esbelta moderó positivamente los efectos del proceso, el } \\
\text { equipo y las relaciones con los proveedores en el desempeño sostenible. }\end{array}$ \\
\hline $\begin{array}{l}\text { Alkhoraif et al., } \\
\text { (2019) }\end{array}$ & $\begin{array}{l}\text { Reino } \\
\text { Unido. }\end{array}$ & $\begin{array}{l}\text { La manufactura esbelta es un método bien establecido de filosofía organizacional que } \\
\text { permite a las empresas mejorar las operaciones de manera más eficaz con mayor } \\
\text { valor y menos desperdicio. Esta investigación ha demostrado la falta de información y } \\
\text { conocimientos relacionados con la implementación de manufactura esbelta en las } \\
\text { Pymes. }\end{array}$ \\
\hline $\begin{array}{l}\text { Nasution et } \\
\text { al., (2018) }\end{array}$ & Indonesia & $\begin{array}{l}\text { La optimización de los recursos (horas hombres, horas máquina, entre otras) se } \\
\text { traducirá en una mayor productividad. }\end{array}$ \\
\hline $\begin{array}{l}\text { Zakaria et al., } \\
(2016)\end{array}$ & Malasia & $\begin{array}{l}\text { Mediante un estudio de simulación tomando como referencia la generación de } \\
\text { desperdicios, se llega a una configuración de planta y de procesamiento que mejora } \\
\text { la productividad. }\end{array}$ \\
\hline
\end{tabular}


Tabla 3: continuación

\begin{tabular}{|l|l|l|}
\hline $\begin{array}{l}\text { Rohani y } \\
\text { Zahraee, } \\
(2015)\end{array}$ & Malasia & $\begin{array}{l}\text { Los resultados finales mostraron que, al implementar algunas técnicas de } \\
\text { pensamiento de manufactura esbelta, el tiempo de producción (PLT) disminuyó de } \\
8,5 \text { días a 6 días, y el tiempo de valor agregado disminuyó de 68 minutos a 37 } \\
\text { minutos. }\end{array}$ \\
\hline $\begin{array}{l}\text { Ahmad et al., } \\
(2017)\end{array}$ & Malasia & $\begin{array}{l}\text { Los resultados finales mostraron que el tiempo total de operación y el tiempo de } \\
\text { actividades de valor no agregado se redujeron con éxito de 1993 segundos a 1719 } \\
\text { segundos y de 234 segundos a 104 segundos, respectivamente. }\end{array}$ \\
\hline $\begin{array}{l}\text { Khusaini et al., } \\
(2016)\end{array}$ & Malasia & $\begin{array}{l}\text { Demuestra que el problema con las organizaciones de la industria de alimentos y } \\
\text { bebidas de Malasia para implementar Manufactura esbelta está básicamente en la } \\
\text { mentalidad, de ahí la percepción. }\end{array}$ \\
\hline $\begin{array}{l}\text { Mafra y Dos- } \\
\text { Santos, (2014) }\end{array}$ & Brasil & $\begin{array}{l}\text { La investigación presentó resultados de mejora de la productividad que confirman } \\
\text { que la aplicación de conceptos de manufactura esbelta puede contribuir } \\
\text { significativamente a reducir los costos de producción. }\end{array}$ \\
\hline $\begin{array}{l}\text { Ferreira et al., } \\
(2017)\end{array}$ & Brasil & $\begin{array}{l}\text { La panadería que aplicó las herramientas de manufactura esbelta redujo los costos } \\
\text { operacionales y aumentó la ganancia neta en alrededor de 25,96\%, aumentando su } \\
\text { índice de margen de ganancia de 12.07\% a 15.33\%. }\end{array}$ \\
\hline
\end{tabular}

Adicional a lo anterior, en referencia a los principales resultados de los documentos de la Tabla 3, los más frecuentes son aquellos que apuntan al aumento de las eficiencias de los procesos productivos, ya que, se muestran impactos palpables en indicadores de procesos como eliminación de residuos, tiempos de ciclo, aumento de rendimientos, continuidad en producción, costos, calidad, vida útil de la maquinaria, disminución de la capacidad ociosa, horas hombre y horas máquina. De igual forma, hay trabajos que apuntan a evaluar qué prácticas de manufactura esbelta aplican y entre las más implementadas se tienen Poka Yoke, Kaizen, 5'S, Kanban y TPM. Las que menos se aplican son: SMED, JIT, Células de Manufactura, Heijunka y Andon. Cabe resaltar, que se viene asociando estos temas con aspectos como el desarrollo sostenible e impacto social, porque son cada vez más frecuentes en la literatura científica y abre paso a temas más mitigación de impacto ambiental y de generación de modelos de negocio más ecológicos. También, es importante resaltar que las empresas pequeñas y medianas tienen muchas barreras en la aplicación de las prácticas de manufactura esbelta y las más frecuentes son la mentalidad y la resistencia al cambio.

Esta revisión sistemática buscó consolidar los parámetros existentes en torno a aplicación de herramientas de manufactura esbelta en la industria panadera para corroborar su adecuación y las implicaciones que conllevan esta herramienta en la reducción de los desperdicios (actividades, tareas, procesos, y demás), así como la evolución que ha tenido a lo largo de los años, tomando como antecedentes los trabajos inmersos en la presente investigación. Las tendencias encontradas muestran el impacto positivo que genera la implementación de esta herramienta en este sector.

A pesar de ser una herramienta que va en crecimiento, en el sector evaluado se observa un bajo nivel de implementación de técnicas de manufactura esbelta debido a que las empresas simplemente se enfocan en tener una buena calidad y cumplir con los parámetros legales (Quesada y Arrieta, 2019), dejando de lado que pueden cumplir con esto aplicando una herramienta de eliminación de desperdicios que ayuda no solo en lo que enfocan sus esfuerzos, sino en la productividad y rentabilidad de la empresa. De los 22 artículos que se tomaron para la presente revisión sistemática, tan solo 3 hacen parte directamente de la industria panadera objeto de estudio. De las tres investigaciones anteriormente mencionadas, los datos obtenidos indican que, aunque la manufactura esbelta aún no es un concepto bien conocido en este segmento de negocios, al aplicar las herramientas se redujeron los costos operacionales y aumentó la ganancia neta (Ferreira et al., 2017). Además, se confirma que la aplicación de conceptos de manufactura esbelta puede contribuir significativamente a reducir los costos de producción (Mafra y Dos-Santos, 2014). De igual modo, las investigaciones descritas en la Tabla 3, avalan la implementación de las herramientas Just In Time (JIT), Total Productive Maintenance (TPM), Single-Minute Exchange of Die (SMED) y 5S (Seiri, Seiton, Seiso, Seiketsu y Shitsuke) propuestas en la presente investigación.

Por otro lado, teniendo en cuenta el crecimiento de la industria de alimentos específicamente en panaderías de Barranquilla y Valledupar, con este trabajo se visualiza que al aplicar herramientas de manufactura esbelta (tales como, Just In Time, SMED, 5S, TPM, Heijunka) se pueden desarrollar acciones de mejora, lo que le permitirá a la empresa implementar los procesos productivos que se están llevando a cabo a nivel mundial, llevándola a estar a la vanguardia en el contexto científico-internacional. De esta manera, este artículo aporta herramientas de reducción de desperdicios que permiten fortalecer las distintas áreas de una empresa (administrativa, operativa, de distribución, entre otras), generando una utilización óptima de recursos, reduciendo costos y generando mayores ganancias, ya que las herramientas analizadas en este documento han sido adoptadas y apropiadas de acuerdo con las necesidades de la industria $\mathrm{y} / \mathrm{o}$ del sector. Adicionalmente, a partir de la revisión de literatura desarrollada en el presente artículo se propone un marco de referencia que sintetiza las tendencias recientes del conocimiento sobre el tema en la Figura 3. 


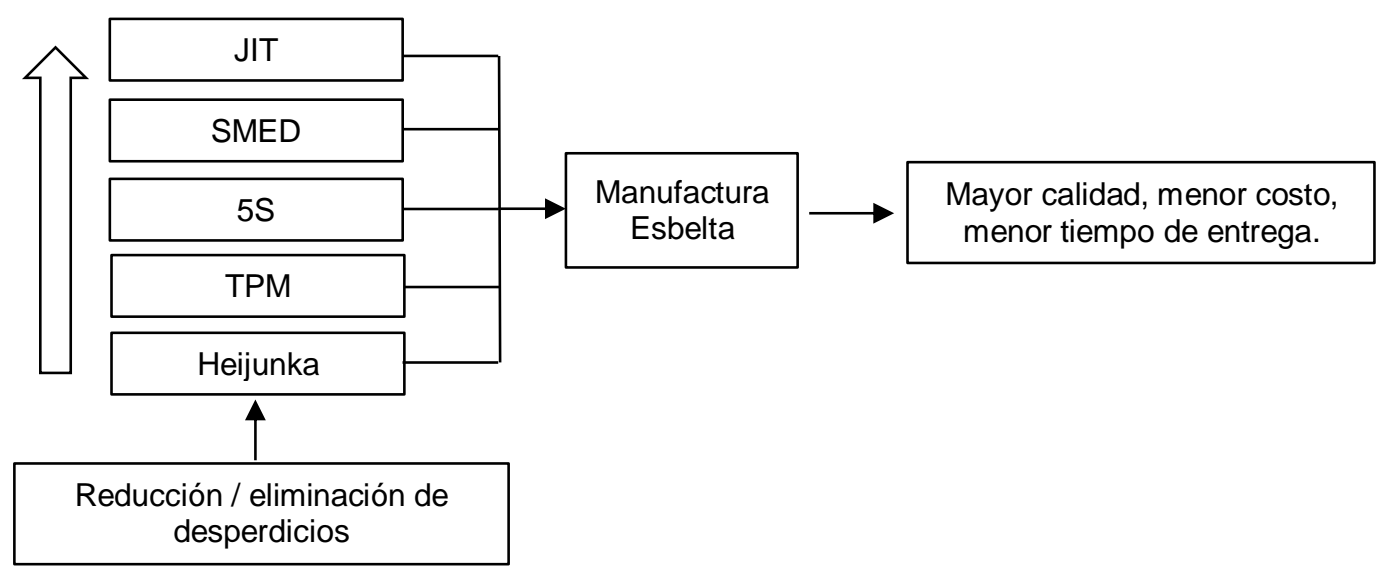

Fig. 3. Marco de referencia de manufactura esbelta dentro del sector panadero de Barranquilla y Valledupar

\section{CONCLUSIONES}

De los resultados logrados y las discusiones establecidas se pueden obtener las siguientes conclusiones: Al analizar los documentos escritos entre los años 2015 y 2019 se evidencio un alza significativa en la literatura científica enfocada a Manufactura esbelta lo que demuestra la importancia de este tema en el contexto científico; de acuerdo a la participación por base de datos, se determinó que Science Reseach tiene el $47 \%$ de los trabajos ; el $36 \%$ de los documentos seleccionados son de países de oriente como Indonesia y Malasia, el $10 \%$ restante proviene de Qatar y Turquía, que son países de oriente medio que viven un gran auge económico en el sector de alimentos y bebidas por su diversificación en la inversión de capitales.

En referencia a los resultados en que se enfocan cada uno de los documentos, los más frecuentes son aquellos que apuntan al aumento de las eficiencias de los procesos productivos, ya que, muestran impactos palpables en indicadores de procesos como: eliminación de residuos, tiempos de ciclo, aumento de rendimientos, continuidad en producción, costos, calidad, vida útil de la maquinaria, disminución de la capacidad ociosa, horas hombre y horas máquina. De igual manera, hay trabajos que apuntan a evaluar qué prácticas de manufactura esbelta aplican y entre las más implementadas se obtienen Poka Yoke, Kaizen, 5'S, Kanban y TPM. De otro lado, del total de palabras de los artículos analizados; cinco de estos fueron las palabras claves con mayor fuerza de enlace total de coincidencia: Lean Manufacturing, literatura review, waste, productivity y bakery. La relación que existe entre manufactura esbelta con las panaderías (bakery), establece que hace poco se está implementado este concepto en las industrias panaderas.

\section{REFERENCIAS}

Ahmad, A. N. A., Lee, T. C. y otros 4 autores, Value stream mapping to improve workplace to support lean environment, doi.org/10.1051/matecconf/201713500032, MATEC Web of Conferences, 135, 1-11 (2017)

Alkhoraif, A., Rashid, H. y McLaughlin, P., Lean implementation in small and medium enterprises: literature review, doi.org/10.1016/j.orp.2018.100089, Operations Research Perspectives, 1-19 (2019)

Antosz, K., y Stadnicka, D., Lean philosophy implementation in SMEs - study results, doi.org/10.1016/j.proeng.2017.03.107, Procedia Engineering, 182, 25-32 (2017)

Arslankaya, S., y Atay, H., Maintenance management and lean manufacturing practices in a firm which produces dairy products, doi.org/10.1016/j.sbspro.2015.10.090, Procedia - Social and Behavioral Sciences, 207, 214-224 (2015)

Baena, C., Entrambasaguas, G. y otros 3 autores, Guía lean manufacturing, 1-66, Instituto Andaluz de Tecnología, Sevilla, España (2008)

Bodek, N., Kaikaku: The power and magic of lean: a study in knowledge transfer, PCS Press Inc., Vancouver, Canada (2010)

Borges-Lopes, R., Freitas, F. y Sousa, I., Application of lean manufacturing tools in the food and beverage industries, doi.org/10.4067/s0718-27242015000300013, Journal of Technology Management and Innovation, 10(3), 120-130 (2015)

Cámara de Comercio de Barranquilla, Base de datos de panaderías legalmente constituidas, (2019)

Cámara de Comercio de Valledupar, Base de datos de panaderías legalmente constituidas, (2019)

Chase, R., Jacobs, R., Aquilano, N., Administración de operaciones producción y cadena de suministros, McGraw-Hill. México DF. (2009)

Consejo Privado de Competitividad, Productividad. La clave del crecimiento para Colombia, ISBN: 978-958-98481-6-6, Zetta Comunicadores, Bogotá, Colombia (2017)

Costa, L. B. M., Godinho-Filho, M., y otros 2 autores, Lean, six sigma and lean six sigma in the food industry: a systematic literature review, doi.org/10.1016/J.TIFS.2018.10.002, Trends in Food Science \& Technology, 82, 122-133 (2018) 
CVN, Industria alimentaria, Centro Virtual de Negocios - Superintendencia de Industria y Comercio SIC, (2018)

Ferreira, W. de P., da-Silva, A. M. y otros 2 autores, Applicability of the lean thinking in bakeries, ISSN: 0798-1015, Revista Espacios, 38(2), 1-11 (2017)

González-Correa, F., Manufactura esbelta (lean manufacturing). Principales herramientas, Revista Panorama Administrativo, 1(2) 85-112 (2007)

Hartini, S., y Ciptomulyono, U., The relationship between lean and sustainable manufacturing on performance: literature review, doi.org/10.1016/j.promfg.2015.11.012, Procedia Manufacturing, 4(2015), 38-45 (2015)

Hernández, C., Así van las MiPymes de la región, Instituto Nacional de Contadores Públicos, (2018)

Iranmanesh, M., Zailani, S. y otros 3 autores, Impact of lean manufacturing practices on firms' sustainable performance: lean culture as a moderator, doi.org/10.3390/su11041112, Sustainability, 11(4), 1-20 (2019)

Khusaini, N. S., Ismail, A. y Rashid, A. A., Investigation of the prominent barriers to lean manufacturing implementation in Malaysian food and beverages industry using Rasch model, doi.org/10.1088/1757-899X/114/1/012090, IOP Conference Series: Materials Science and Engineering, 114(1), 1-9 (2016)

Kumar, S. A., y Suresh, N., Operations Management, New Age International, New Delhi (2009)

Mafra, R., y Dos-Santos, A. J., Aplicação de conceitos de manufatura enxuta na indústria de panificação e confeitaria: caso de pequena empresa de panificação de Joinville, Brasil, ISSN: 0798-1015, Revista Espacios, 36(1), 1-12 (2015)

Moeuf, A., Tamayo, S. y otros 3 autores, Strengths and weaknesses of small and medium sized enterprises regarding the implementation of lean manufacturing, doi.org/10.1016/j.ifacol.2016.07.552, IFAC-PapersOnLine, 49(12), 71-76 (2016)

Munteanu, V., y Ştefănigă, A., Lean manufacturing in SMEs in Romania, doi.org/10.1016/j.sbspro.2018.04.028, Procedia - Social and Behavioral Sciences, 238(004), 492-500 (2018)

Nasution, A. A., Siregar, I. y otros 4 autores, Lean manufacturing applications in the manufacturing industry, doi.org/10.1051/matecconf/201822002005, MATEC Web of Conferences, 220, 1-5 (2018)

Padilla, L., Lean manufacturing - Manufactura esbelta/ágil, ISSN: 2076-3166, Revista Electrónica Ingeniería Primero, 15(15), 64-69 (2010)

Psomas, E., y Antony, J., Research gaps in lean manufacturing: a systematic literature review, doi.org/10.1108/IJQRM12-2017-0260, International Journal of Quality and Reliability Management, 36(5), 815-839 (2019)

Quesada, M. del R., y Arrieta, J. G., Implementation of lean manufacturing techniques in the bakery industry in Medellin, doi.org/10.1590/0104-530x-2505-19, Gestão \& Produção, 26(2), 1-9 (2019)

Rajadell-Carreras, M., y Sánchez, J. L., Lean manufacturing: la evidencia de una necesidad, 1a Ed., Ediciones Díaz de Santos, Madrid, España (2010)

Rohani, J. M. y Zahraee, S. M., Production line analysis via value stream mapping: a lean manufacturing process of color industry, doi.org/10.1016/j.promfg.2015.07.002, Procedia Manufacturing, 2, 6-10 (2015)

Salem, R., Musharavati, F., y otros 2 autores, An empirical study on lean awareness and potential for lean implementations in Qatar industries, doi.org/10.1007/s00170-015-7421-7, International Journal of Advanced Manufacturing Technology, 82, 1607-1625 (2016)

Sarria, M. P., Fonseca, G. A. y Bocanegra-Herrera, C. C., Modelo metodológico de implementación de lean manufacturing, doi.org/10.21158/01208160.n83.2017.1825, Revista EAN, 83, 51-71 (2017)

Socconini, L., Lean manufacturing paso a paso, 1-368, Grupo Editorial Norma, (2008)

Sousa, E., Silva, F. J. G. y otros 4 autores, Applying SMED methodology in cork stoppers production, doi.org/10.1016/j.promfg.2018.10.103, Procedia Manufacturing, 17, 611-622 (2018)

Stevenson, L., Jain, R., Lean manufacturing in the food industry, ISSN: 1475-3324, Food, Science and Technology, 19(3), 32-34 (2005)

Tapia, J., Escobedo, T. y otros 3 autores, Marco de referencia de la aplicación de manufactura esbelta en la industria, doi.org/10.4067/S0718-24492017000300171, Ciencia \& Trabajo, 19(60), 171-178 (2017)

Velásquez, L., Estudio del alcance de la implantación de tecnologías de información, como apoyo al mejoramiento de los procesos, en las pequeñas y medianas empresas del sector manufacturero en Bogotá, Pont. Univ. Javeriana, Bogotá DC. (2003)

Viteri, J., Matute, E. y otros 2 autores, Implementation of lean manufacturing in a food enterprise, ISSN: 1390-6542, Enfoque UTE, 7(1), 1-12 (2016)

Widiasih, W., Karningsih, P. D., y Ciptomulyono, U., Development of integrated model for managing risk in lean manufacturing implementation: a case study in an Indonesian manufacturing company, doi.org/10.1016/j.promfg.2015.11.042, Procedia Manufacturing, 4, 282-290 (2015)

Womack, J.P., Jones, D.T., Roos, D., The machine that changed the world, Rawson Associates, New York, USA (1990)

Zakaria, N. H., Nik-Mohamed, N. M. Z. y otros 2 autores, Lean manufacturing implementation in reducing waste for electronic assembly line, doi.org/10.1051/matecconf/20179001048, MATEC Web of Conferences, 90, 1-10 (2016) 
